\title{
Neuron absorption study and mid-IR optical excitations
}

Dingkai Guo, Xing Chen, Shilpa Vadala, Jennie Leach, Yordan Kostov, et al.

Dingkai Guo, Xing Chen, Shilpa Vadala, Jennie Leach, Yordan Kostov, William W. Bewley, Chul-Soo Kim, Mijin Kim, Chadwick L. Canedy, Charles D. Merritt, Igor Vurgaftman, Jerry R. Meyer, Fow-Sen Choa, "Neuron absorption study and mid-IR optical excitations," Proc. SPIE 8207, Photonic Therapeutics and Diagnostics VIII, 820750 (9 February 2012); doi: 10.1117/12.909561

SPIE. Event: SPIE BiOS, 2012, San Francisco, California, United States 


\title{
Neuron Absorption Study and Mid-IR Optical Excitations
}

\author{
Dingkai Guo*a, Xing Chen ${ }^{\mathrm{a}}$, Shilpa Vadala ${ }^{\mathrm{a}}$, Jennie Leach ${ }^{\mathrm{a}}$, Yordan Kostov ${ }^{\mathrm{a}}$, William W. Bewley ${ }^{\mathrm{b}}$ \\ Chul-Soo Kim ${ }^{\mathrm{b}}$, Mijin Kim ${ }^{\mathrm{b}}$, Chadwick L. Canedy ${ }^{\mathrm{b}}$, Charles D. Merritt ${ }^{\mathrm{b}}$, Igor Vurgaftman ${ }^{\mathrm{b}}$, Jerry R. \\ Meyer ${ }^{\mathrm{b}}$, and Fow-Sen Choa ${ }^{\mathrm{a}}$ \\ ${ }^{a}$ University of Maryland, Baltimore County, 1000 Hilltop Circle, Baltimore, MD USA 21250; \\ ${ }^{\mathrm{b}}$ Code 5613, Naval Research Laboratory, Washington, DC USA 20375 \\ *dingk1@umbc.edu; phone 1443 682-5166; fax 1410 455-6500
}

\begin{abstract}
Neuronal optical excitation can provide non-contacting tools to explore brain circuitry and a durable stimulation interface for cardiac pacing and visual as well as auditory sensory neuronal stimulation. To obtain accurate absorption spectra, we scan the transmission of neurons in cell culture medium, and normalize it by subtracting out the absorption spectrum of the medium alone. The resulting spectra show that the main neuronal absorption peaks are in the 3000$6000 \mathrm{~nm}$ band, although there is a smaller peak near $1450 \mathrm{~nm}$. By coupling the output of a $3 \mu \mathrm{m}$ interband cascade laser (ICL) into a mid-IR fluorozirconate fiber, we can effectively deliver more than $1 \mathrm{~J} / \mathrm{cm}^{2}$ photon intensity to the excitation site for neuronal stimulation.
\end{abstract}

Keywords: mid-infrared, neuron optical excitation

\section{INTRODUCTION}

Neuronal optical excitation can provide non-contacting tools to explore brain circuitry and also a durable stimulation interface for cardiac pacing and visual as well as auditory sensory neuronal stimulation. Laser inhibition and stimulation of neurons have been reported previously using visible or UV photons ${ }^{1,2}$. Recent techniques like binding photosensitive molecules to assist the excitation ${ }^{3}$ and using genetically altered cells ${ }^{4}$ to depolarize the neuron and evoke action potentials were also applied to stimulate neurons. However, these approaches either involve photochemical reactions that cause cell damage/death or they are not yet practical for clinical application due to the possibility of introducing foreign materials into human bodies during the procedure.

Recent studies have shown it will be much safer to excite neurons through a thermal process, which uses heat to open the nerve cell's ion exchange channels and evoke action potentials ${ }^{5,6}$. They found that the wavelength selection is critical, and that longer wavelengths of $1.45-1.54 \mu \mathrm{m}$ and near $2 \mu \mathrm{m}$ range more easily depolarize the cell.

With the recent development of high-power mid-IR (MIR) semiconductor lasers, including quantum cascade lasers ${ }^{7}$ (QCLs), interband cascade lasers ${ }^{8,9}$ (ICLs), and $\mathrm{Sb}$ based interband diodes ${ }^{10}$, convenient sources can now cover wavelengths all the way from 2 to $20 \mu \mathrm{m}$. Selecting the right excitation wavelength can effectively stimulate neurons with the lowest level of peak power and pulse energy. Portable and battery supported optical stimulation units can then be developed for applications as heart pacemakers, and visual and auditory assist devices for aging patients with degenerated sensory function.

\section{EXPERIMENTS}

To obtain accurate absorption spectra of living neurons, we must scan the neuron transmission when they are in a serumcontaining cell culture medium, and then normalize it by subtracting out the absorption spectrum of the medium alone with the same optical setup. To achieve that we use GaAs wafers for the windows of the chamber containing the serum and neurons. Although we tried to use silicon and considered other MIR window materials, Fig.1 shows that the MIR transmission spectrum of silicon is not flat and its transmission is lower. Other windows are either water soluble or not bio-compatible. The GaAs wafer is seen to have its highest transmission near $\lambda=18 \mu \mathrm{m}$. A very thin $(100 \mathrm{~nm}) \mathrm{SiO}_{2}$ layer was grown on top of the GaAs substrate to protect the confined neuron cells. Figure 2 shows that the thin $\mathrm{SiO}_{2}$

Photonic Therapeutics and Diagnostics VIII, edited by Nikiforos Kollias, et al., Proc. of SPIE Vol. 8207, 820750

(c) 2012 SPIE · CCC code: 1605-7422/12/\$18 $\cdot$ doi: 10.1117/12.909561 
layer induces a small absorption dip in the MIR transmission spectrum at $9.5 \mu \mathrm{m}$, but does not have much effect on the overall spectrum. The cell culture device is illustrated schematically in Fig. 3(a). Two cell culture devices with the same dimensions were fabricated; one containing the neuron cells in medium while the other contained only the medium for optical background subtraction. The cultured rat neurons (PC12 cell line, ATCC) in medium were imaged by phase microscopy in the upper panel of Fig.3 (b). To minimize water absorption in the MIR region, the measurement was done immediately before the medium dehydrated. The lower panel shows the neurons following complete dehydration.

Figure 4 shows the resulting normalized absorption spectrum for the cultured neurons. The main neuron absorption peaks are seen to be close to $3000 \mathrm{~nm}$ and $6000 \mathrm{~nm}$. A lower and broader absorption region is also shown between 1450 $2600 \mathrm{~nm}$. These features are consistent with the large percentage of water contained in the neuron cells, which will make it more difficult to isolate the heating process since the neurons are surrounded by aqueous medium. In fact, the absorption of optical excitation by nearby water molecules can evoke action potential. However, the spatial resolution of such neuron stimulation is low so it requires high excitation pulse energy. The small difference between the thresholds for stimulation and damage can potentially make operations more dangerous. Furthermore, heating a larger amount of mass lengthens the cooling time, forcing a reduction of the laser pulse repetition rate. Therefore, an MIR fiber must be used to deliver photons from the MIR source to the excitation site.

To effectively excite the neurons we choose to use $3 \mu \mathrm{m}$ photons provided by a broad area interband cascade laser ${ }^{8}$ (ICL). With an injection current of $950 \mathrm{~mA}$ this mid-IR source lases at $3160 \mathrm{~nm}$, which shifts to $3265 \mathrm{~nm}$ for operation at 1.25A. Both wavelengths are inside the broad neuron absorption peak near $3 \mu \mathrm{m}$, as shown in the inset of Fig.4. The laser was then put into a Liquid Nitrogen dewar and cooled to 77K. The LIV curves and lasing spectra measured in pulsed mode are shown in Fig.5. The wavelength shifted to around $2.9 \mu \mathrm{m}$ however, it was still inside the big neuron absorption peak near $3 \mu \mathrm{m}$.

Two mainstream fibers are available for photon delivery in the MIR range. Chalcogenide IR (CIR) fibers are unsuitable, however, because the core material (typically $\mathrm{As}_{2} \mathrm{~S}_{3}$ ) is toxic to neuron cells. Fluoride fibers are also having issues since the main component, Fluorozirconate $\mathrm{ZrF}_{4}$, becomes water soluble at $\mathrm{pH}$ values slightly lower than 7 . Fortunately physiological $\mathrm{pH}$ is $\sim 7.3$ and adding an appropriate coating as a protection allowed us to proceed with a $1 \mathrm{~m}$ long Fluoride fiber in the experiment. The spectrum in Fig. 6 indicates that this Fluoride fiber has very low absorption at 3 $\mu \mathrm{m}$. Figure 7 shows the light coupling scheme from the ICL to the MIR fiber. The optimized coupling efficiency for the direct coupling method reaches $25 \%$, as indicated by the plots of laser output and fiber output $v$. operating current shown in Fig.8.

The cultured neuron cells were mounted on a circuit board for excitation as shown in Fig. 9 (a). Figure 9 (b) shows the neuron firing signals induced by touching the top contact electrodes with a fixed amount of electrical potential, which generated a train series of excitation pulses and some of them can cause 1-2 followed neuron firing pulses right after the external stimulations. The equivalent circuit in Fig. 9(c) shows the nerve cells lying in between the top and bottom electrodes like a leaky capacitor $(\mathrm{R} / / \mathrm{C})$ in parallel with an electrical source. Any charge build-up caused by the electrical excitation will create a potential spike that eventually discharges to a low potential through the resistor. The study neuron firing induced by MIR light is in progress.

\section{CONCLUSIONS}

In this study, we identified MIR absorption peaks for cultured rat neurons at $3000 \mathrm{~nm}$ and $6000 \mathrm{~nm}$ region, as well as the 1450- $2600 \mathrm{~nm}$ region. We are now using interband cascade laser emission via an MIR fiber to deliver pulsed MIR excitation light to the neuron cell site.

\section{REFERENCES}

[1] J. E. Olson, W. Schimmerling, G. C. Gundy, and C. A. Tobias, "Laser microirradiation of cerebellar neurons in culture, Electrophysiological and morphological effects," Cell Biophys. 3, 349 (1981).

[2] I. C. Farber and A. Grinvald, "Identification of presynaptic neurons by laser photostimulation," Science 222, 1025 (1983). 
[3] H. Wang, J. Peca, M. Matsuzaki, K. Matsuzaki, J. Noguchi, L. Qiu, D.Wang, F. Zhang, E. Boyden, K. Deisseroth, H. Kasai, W. C. Hall, G. Feng, and G. J. Augustine, "High-speed mapping of synaptic connectivity using photostimulation in Channelrhodopsin-2 transgenic mice," Proc. Natl. Acad. Sci. USA 104, 8143 (2007).

[4] Gottschalk, and K. Deisseroth, "Multimodal fast optical interrogation of neural circuitry," Nature 446, 633 (2007).

[5] J. Wells, C. Kao, K. Mariappan, J Albea, ED Jansen, P. Konrad, A. Mahadevan-Jansen, "Optical stimulation of neural tissue in vivo," Opt Lett. 30, 504 (2005).

[6] R. G. McCaughey, C. Chlebicki, and B. J.Wong, "Novel wavelengths for laser nerve stimulation," Laser Surg. Med. 42:69-75 (2010).

[7] See, for example, http://www.daylightsolutions.com/products/lasers/aries.htm : commercially available tunable high power MIR lasers.

[8] I. Vurgaftman, W. Bewley, C. L. Canedy, C. Kim, M. Kim, J. R. Lindle, C. D. Merritt, J. Abell, J. R. Meyer, "MidIR Type-II Interband Cascade Laser," IEEE Journal of Selected Topics in Quantum Electronics, 99, 1 (2011).

[9] I. Vurgaftman, W. W. Bewley, C. L. Canedy, C. S. Kim, M. Kim, C. D. Merritt, J. Abell, J. R. Lindle, and J. R. Meyer, "Rebalancing of Internally Generated Carriers for Mid-IR Interband Cascade Lasers with Very Low Power Consumption," Nature Commun. 2, 585 (2011).

[10] T. Hosoda, G. Kipshidze, L. Shterengas, G. Belenky, "200mW type-I GaSb-based laser diodes operating at $3 \mu \mathrm{m}$ : Role of waveguide width,” Appl. Phys. Lett. 94, 261104 (2009).

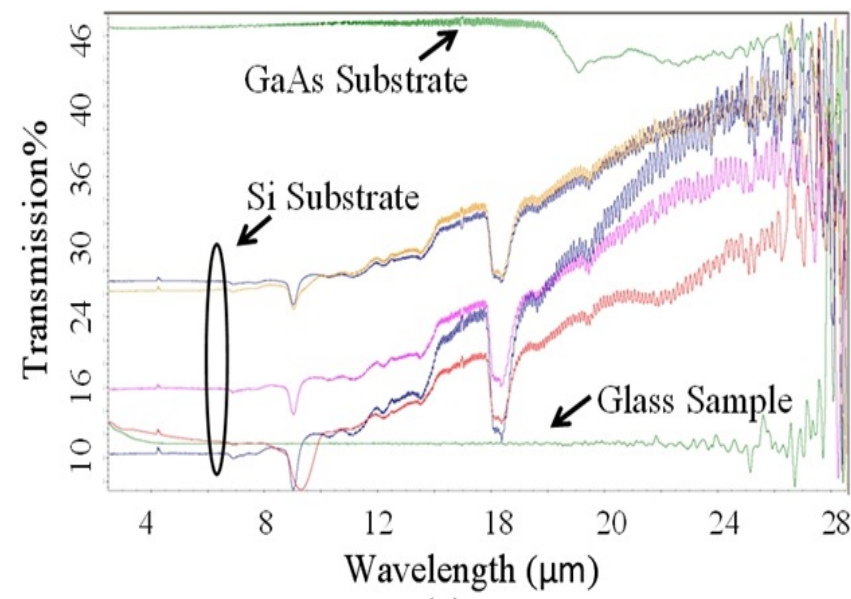

(a)

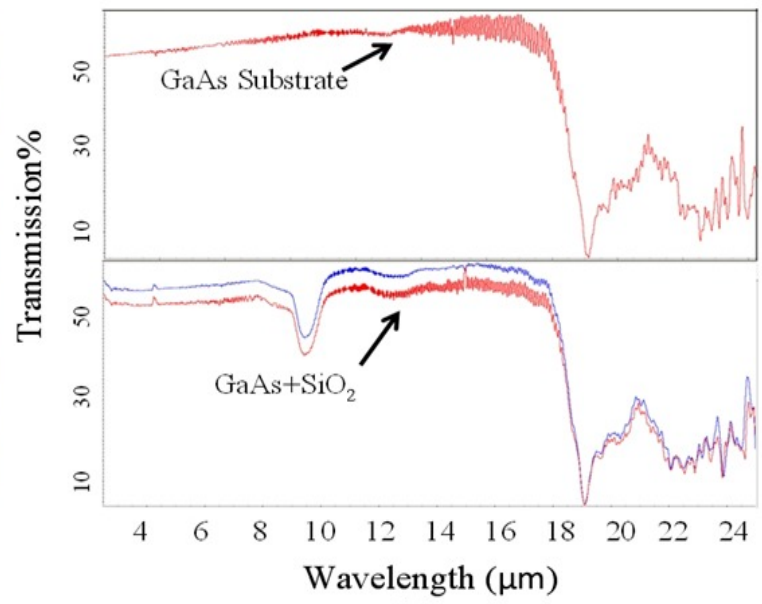

(b)

Figure 1. (a) MIR transmission spectrum of GaAs, Silicon and a glass substrate, (b) MIR transmission spectra of the GaAs substrate alone, and two similar GaAs samples with different thickness and each coated by $100 \mathrm{~nm}$ of $\mathrm{SiO}_{2}$ 

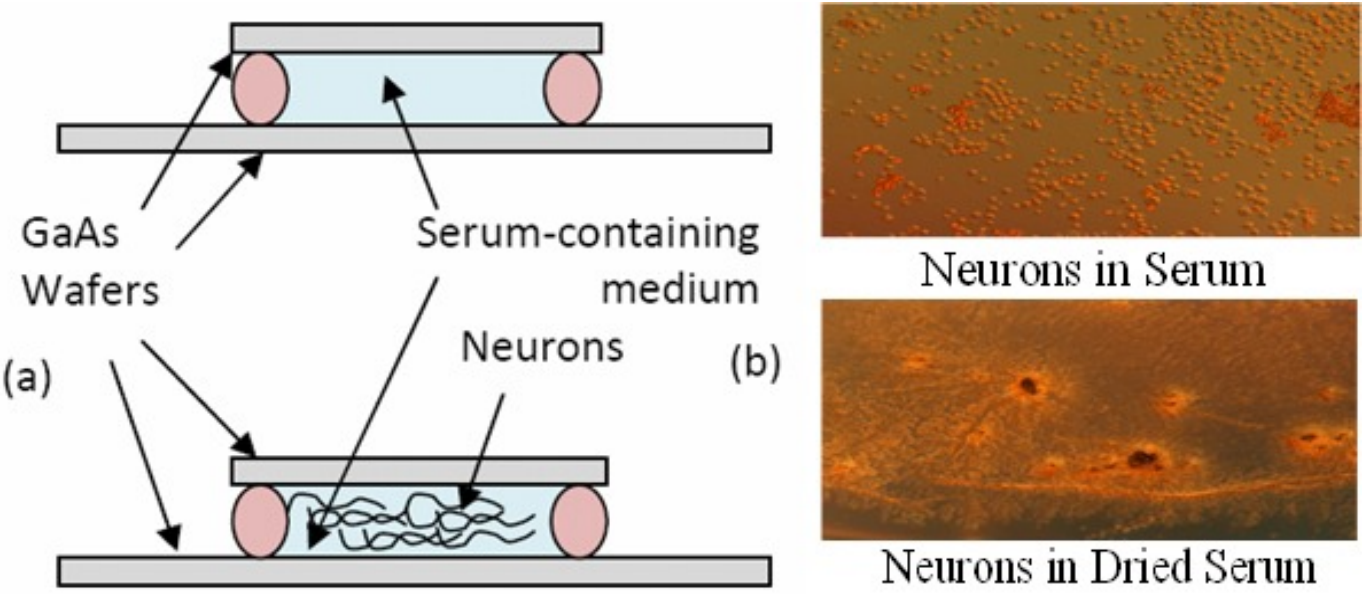

\section{Neurons in Serum}

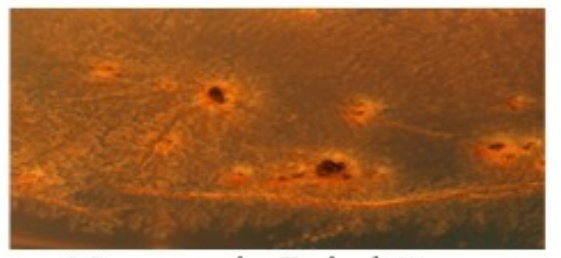

Neurons in Dried Serum

Figure 2. (a) Serum and neuron containers built by using GaAs wafers and rubber sealing gasket. (b) Neuron cells in the cultured medium, before and after dehydration.

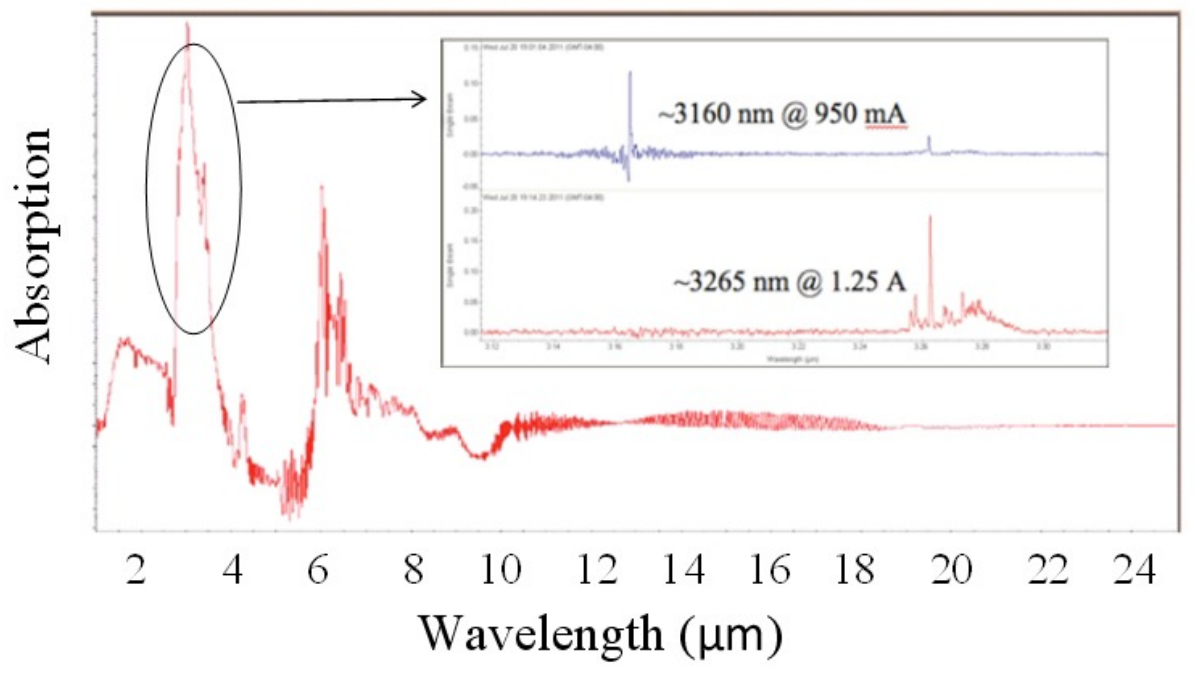

Figure 3. Cultured rat neuron MIR absorption spectrum. Inset: The lasing spectrum of the Interband Cascade Laser at two different operating currents 


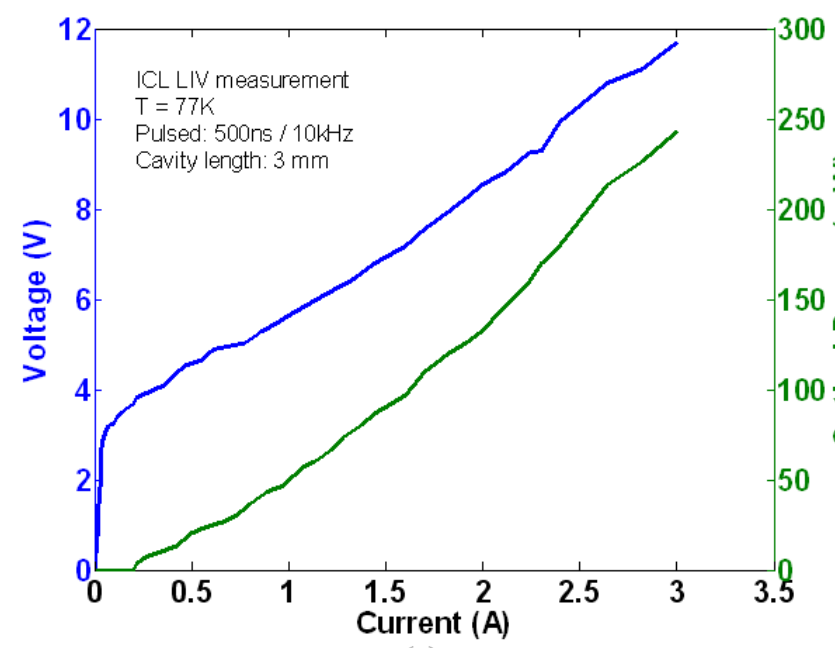

(a)

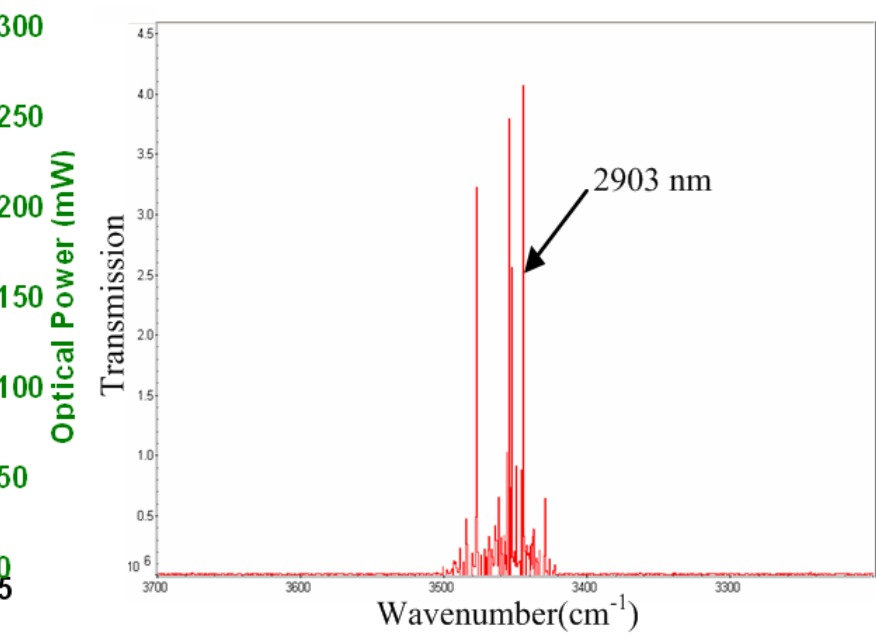

(b)

Figure 4. (a) LIV curve and (b) Lasing spectra of the Interband Cascade Laser operated in pulsed mode at $77 \mathrm{~K}$

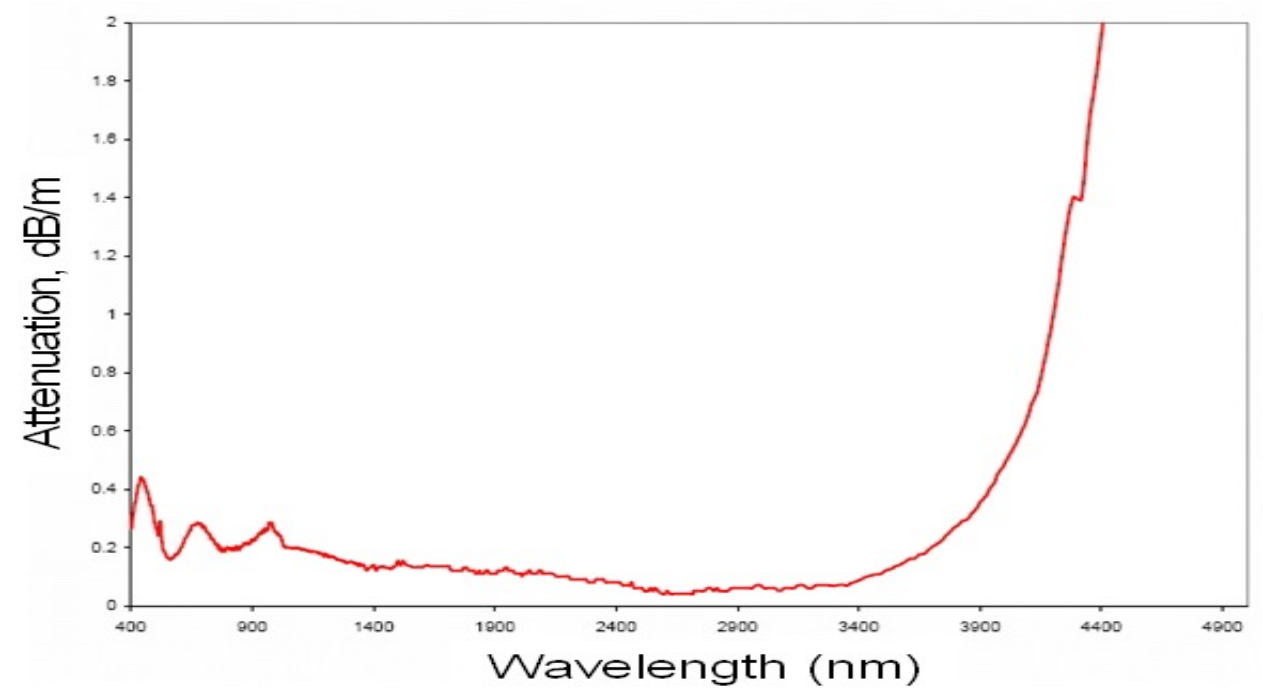

Figure. 5. Absorption spectrum of the 1m-long Fluorozirconate fiber.

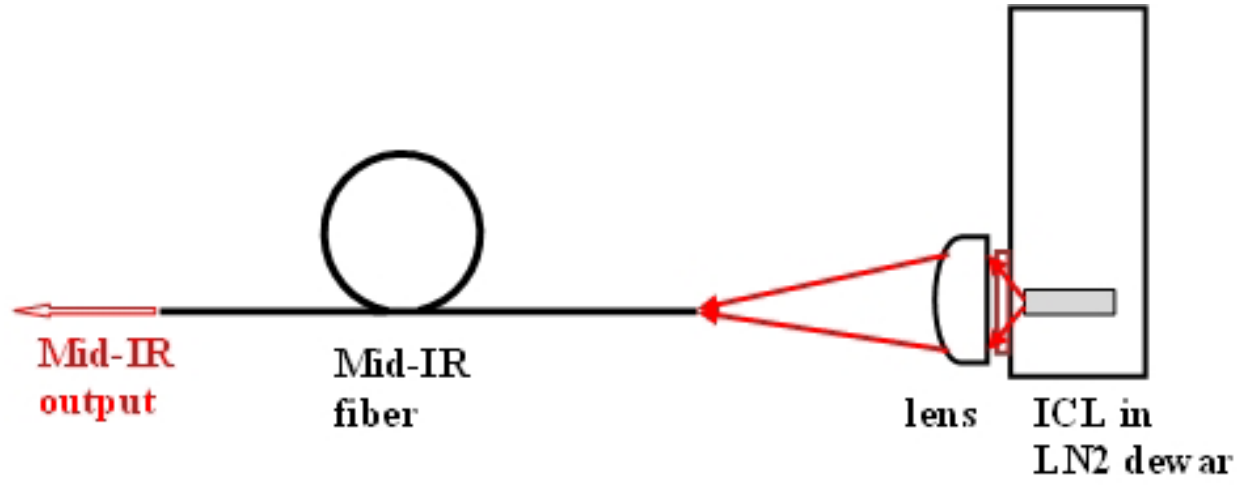

Figure 6. ICL mid-IR fiber coupling schematic 


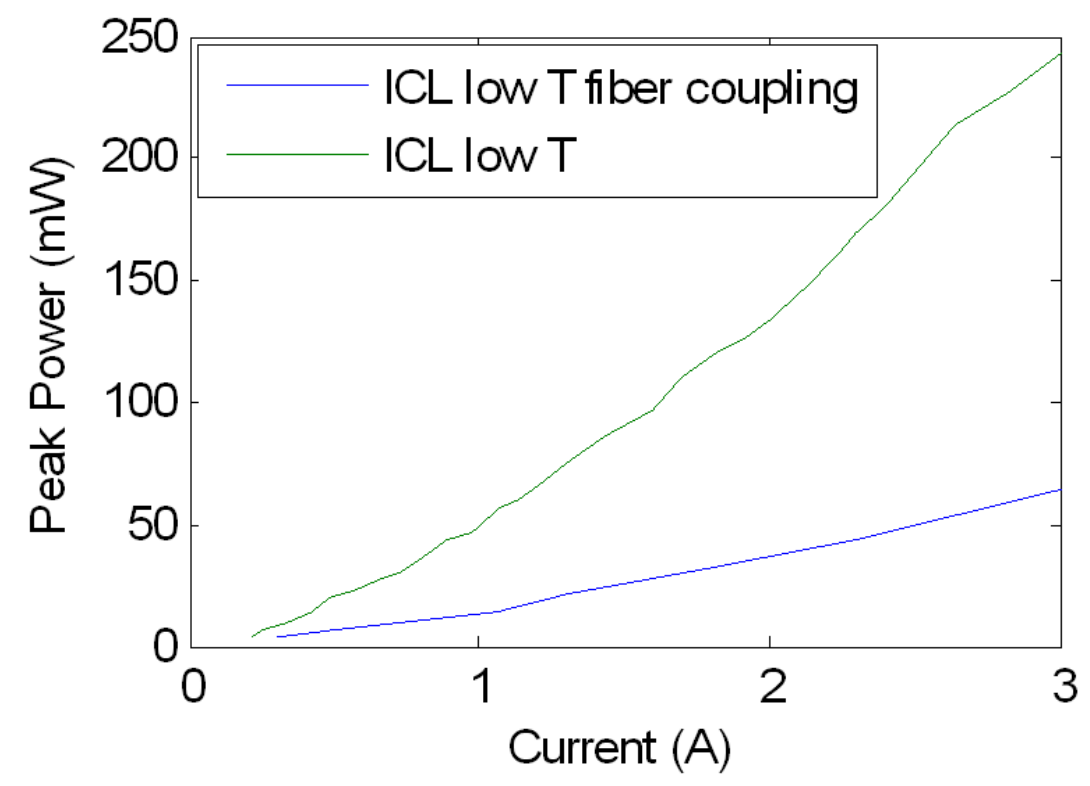

Figure 7. L-I characteristics of the ICL at low temperature (77K) before and after coupling to the MIR fiber. Before fiber coupling the peak power from the ICL is $\sim 250 \mathrm{~mW}$; after coupling, the MIR output from the fiber is $\sim 60 \mathrm{~mW}$, which corresponds to a coupling efficiency of $\sim 25 \%$. The laser was operated with $500 \mathrm{~ns}$ pulses and 10 $\mathrm{kHz}$ repetition rate.
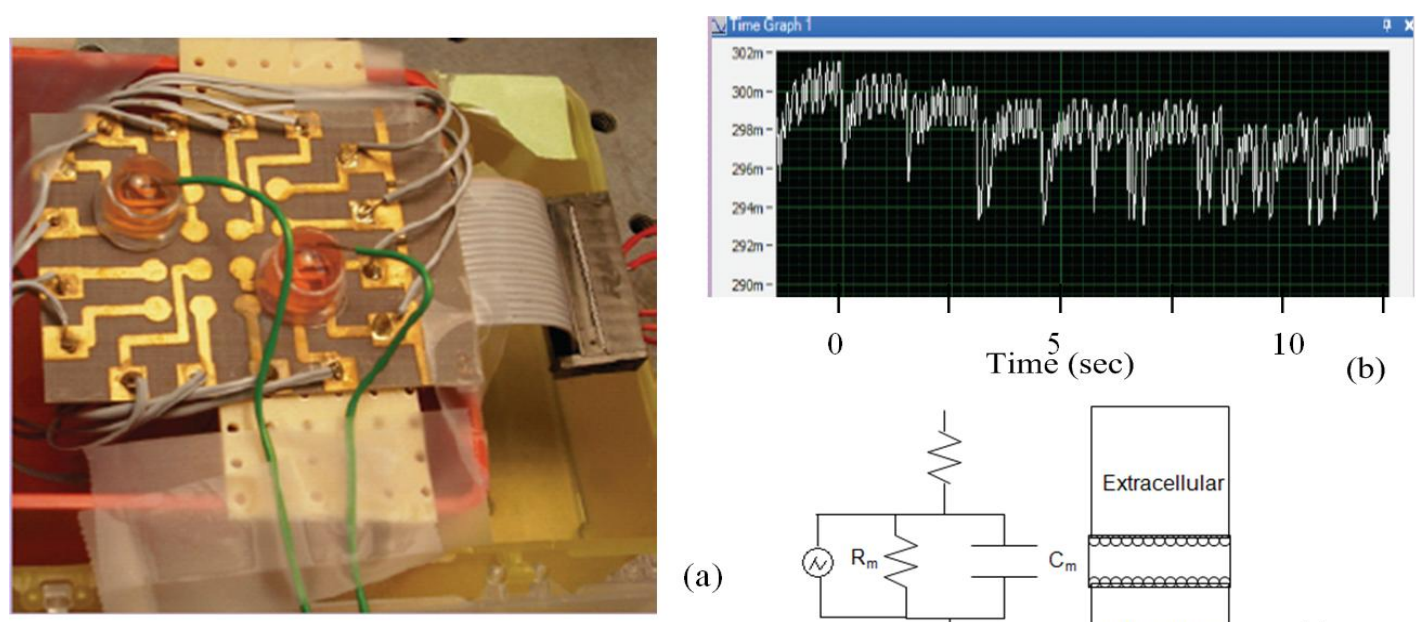

(a)

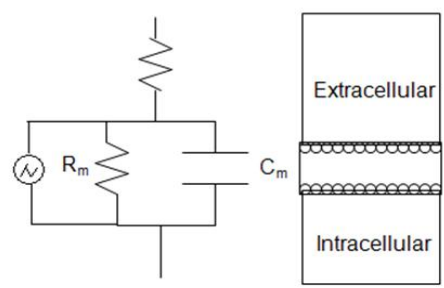

(c)

Figure 8. (a) The cultured neuron cells mounted on a circuit board. (b) The neuron firing signals (double and triple pulses) immediately after external stimulations. (c) The equivalent neuron cell circuitry for the excitation model. 